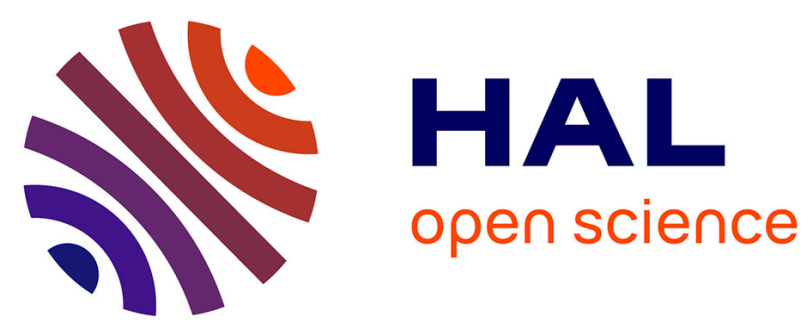

\title{
A Scalable Information Security Technique: Joint Authentication-Coding Mechanism for Multimedia over Heterogeneous Wireless Networks
}

Liang Zhou, Baoyu Zheng, Anne Wei, Benoit Geller, Jingwu Cui

\section{- To cite this version:}

Liang Zhou, Baoyu Zheng, Anne Wei, Benoit Geller, Jingwu Cui. A Scalable Information Security Technique: Joint Authentication-Coding Mechanism for Multimedia over Heterogeneous Wireless Networks. Kluwer Journal of Wireless Personal Communications, 2009, 10.1007/s11277-008-9595-x . hal-01238447

HAL Id: hal-01238447

https://hal-ensta-paris.archives-ouvertes.fr/hal-01238447

Submitted on 4 Dec 2015

HAL is a multi-disciplinary open access archive for the deposit and dissemination of scientific research documents, whether they are published or not. The documents may come from teaching and research institutions in France or abroad, or from public or private research centers.
L'archive ouverte pluridisciplinaire HAL, est destinée au dépôt et à la diffusion de documents scientifiques de niveau recherche, publiés ou non, émanant des établissements d'enseignement et de recherche français ou étrangers, des laboratoires publics ou privés. 


\title{
A Scalable Information Security Technique:
} Joint Authentication-Coding Mechanism for Multimedia over Heterogeneous Wireless Networks

\author{
Liang Zhou, Baoyu Zheng, Anne Wei, Benoît Geller, Jingwu Cui
}

\begin{abstract}
As multimedia is expected to be a major traffic source in the next-generation wireless networks, there have been increasing concerns about the security issues of wireless transmission of multimedia in recent years. Wireless networks, by their natures, are more vulnerable to external intrusions than wired ones. Therefore, many applications demand authenticating the integrity of multimedia content delivered wirelessly. In this work, we propose a framework for jointly authenticating and coding multimedia to be transmitted over heterogeneous wireless networks. We firstly provide a novel graphbased authentication scheme which can not only construct the authentication graph flexibly but also trade-off well among some practical requirements such as overhead, robustness and delay. And then, a rate-distortion optimized joint source-channel coding (JSCC) approach for error-resilient scalable encoded video is presented, in which the video is encoded into multiple independent streams and each stream is assigned forward error correction (FEC) codes to avoid error propagation. Furthermore, we consider integrating authentication with the specific JSCC scheme to achieve a satisfactory authentication results and end-to-end reconstruction quality by optimally applying the appropriate authentication and coding rate. Simulation results show the effectiveness of the proposed authentication-coding scheme for multimedia over wireless networks.
\end{abstract}

\section{Index Terms}

multimedia security; authentication; joint source-channel coding; wireless networks 


\section{INTRODUCTION}

As multimedia is expected to be a major traffic source on the next-generation wireless networks, the demand for transmitting the multimedia content over wireless networks has increased. In contrast to the abundance of methods have been proposed to design robust and efficient schemes for delivering multimedia content over error-prone wireless networks, there are only very few works paying attention to the security aspect of such transmission. In fact, as more and more applications require authenticated multimedia streams, it is important to protect the authenticity of the streams in the aspects of integrity and non-repudiation. In order to design a satisfactory authentication scheme for a wireless multimedia transmission system, it would be essential to take into account the following practical requirements:

- Low communication overhead: It refers to the additional bytes transmitted along with the stream packets. These additional bytes include i.e. MAC (Message Authentication Code), Crypto Hash values or digital signatures.

- Robust against packet loss: The packets of the stream should be able to be authenticated with high probability under varied channel conditions with different packet loss rate. This requirement is particularly useful for multimedia streams which can tolerate some packet loss.

- Less receiver delay: It refers to the delay from the time the packet is received to the time when it is authenticated by the receiver. When consuming streaming media, each packet usually has its deadline after which it becomes useless. As a result, a large receiver delay could cause a packet to miss its deadline.

\section{A. Related Works}

The authentication problem has been attempted mainly using two approaches: a naive solution of authenticating a potential long stream is to sign each network packet using digital signature. However the problem is that signing algorithms nowadays are computationally expensive, and it is not worthy to compute and verify one signature for each packet [1]; since it is too expensive to sign every packet of the stream, we can organize packets into groups and sign only one packet within each group [14]. This approach can be further classified into graph-based approach [2-5] and erasure-code-based approach [6]. [2] proposed an authentication scheme using a simple hash chain. It has low overhead and low receiver delay, but it cannot tolerate any packet loss; [3] provided EMSS, which uses a hash chain where each packet contains the hashes of previous 
packets and the signing is on the last packet. Obviously, it easily leads to a high receiver delay; [4] presentd an authentication scheme based on the expander graph and theoretically derived the lower bound of authentication probability (AP). However, it has a very large communication overhead which is unacceptable for real applications; [5] was based on the random graph. The signing is on the first packet, and each packet contains the hashes of every subsequent packet with certain probability. Therefore, it also has high communication overhead; [6] was proposed to use erasure code for stream authentication. For each block, the digital signature is coded with erasure code and then scattered into the packets. As long as the number of loss packets is less than a threshold, all received packets can be authenticated. This scheme has a high computation overhead due to the erasure coding. In addition, it also suffers from a high receiver delay, because the receiver has to wait for a minimum number of the received packets before authentication.

\section{B. Main Contributions}

The main contributions of this paper are as follows: firstly, we present a novel graph-based authentication (NGBA) approach which can not only construct the authentication graph flexibly but also trade-off well between the aforementioned practical requirements. Secondly, we propose an analytical joint source-channel coding (JSCC) approach for error-resilient scalable encoded video for lossy transmission, in which the video is encoded into multiple independent substreams based on 3-D SPIHT (3-D set partitioning in hierarchical trees) algorithm to avoid error propagation. Furthermore, the final realization of joint authentication-coding (JAC) system is the highlight of the proposed scheme because the ultimate goal of such scheme is to achieve an optimal end-to-end multimedia quality under the overall limited resource budget.

\section{Outline}

The rest of paper is organized as follows. In section II, we provide some technical preliminaries used in this work. Section III introduces the novel graph-based authentication scheme and joint source-channel coding, respectively. In section IV, we optimize the proposed joint authenticationcoding scheme. Finally, we present some selected simulation results and give some concluding remarks. 


\section{PRELIMINARIES}

\section{A. Definitions and Notations}

Considering a sender transmitting consecutive packets $\left\{P_{1}, \ldots, P_{n}\right\}$ in a broadcast data stream, we construct an authentication graph (AG) to authenticate received packets. In particular, we construct a directed acyclic graph of $n$ vertices where a vertex $i$ corresponds to the packet $P_{i}$. Let $e(i, j)$ denotes a directed edge starting from $i$ and ending at $j$. An edge $e(i, j)$ in the graph indicates the authentication relationship between packet $P_{i}$ and $P_{j}$ : upon receiving packet $P_{i}$ and $P_{j}$, if a receiver can authenticate both the contents and the source of $P_{i}$, then it can authenticate the contents and source of $P_{j}$. One of the packets, denoted by $P_{s i g}$, is signed with a public key signature algorithm. Hence, packet $P_{i}$ can be authenticated if and only if there is a path from $P_{i}$ to the signature packet that only includes nodes corresponding to the received packets [7]. We denote the probability that $P_{i}$ is linked to $P_{s i g}$ via such a path by $\operatorname{Pr}\left[P_{i} \rightarrow P_{s i g}\right]$.

For every stream, we are interested in the value of $\operatorname{Pr} r_{i}=\operatorname{Pr}\left[P_{i} \rightarrow P_{\text {sig }} \mid P_{i}\right.$ is received $]$ for $i \in 1, \ldots, n$. In particular, we allow the sender to input desired values for these authentication probabilities. It is useful to allow a different AP for each packet, because the packets in the stream may actually vary in priority. Consequently, packets deemed more important will be

more tolerant to loss (because redundant authentication information will be included), and the less important packets will be less tolerant of loss, in order to avoid unnecessary overhead.

\section{B. Hash-Based Authentication Tool}

A public hash function may be used to link the packets in a multicast stream to a signature. Recall from subsection II-A that $e(i, j)$ is representing in a graph, then in the corresponding authentication scheme, the ability to authenticate $P_{j}$ implies the ability to authenticate $P_{i} . P_{i}$ may have a positive in-degree itself, indicating that hashes of other packets are included within $P_{i}$. In this case, the hash of $P_{i}$ is taken after all other hashes it requires are included in it. We require strictly hash-based authentication graphs to be acyclic, so as to avoid dependencies between packets which can not be fulfilled [8].

One major advantage of this signature-based approach is that it can protect data integrity while ensuring non-repudiation. Therefore, it is useful for general authentication applications when digital evidence is concerned. Other merits of this approach include achieving both low 


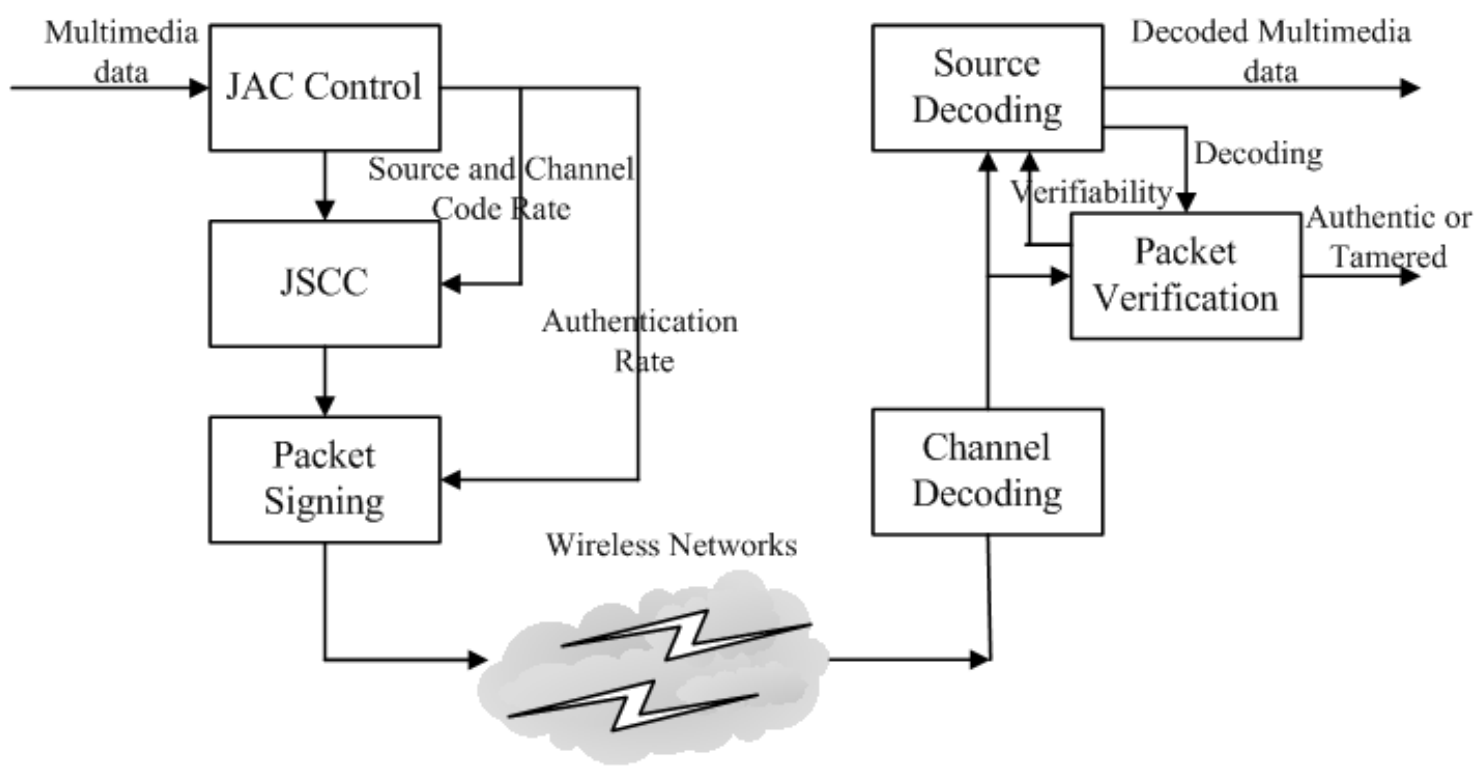

Fig. 1. Architecture of the joint authentication and coding scheme.

computation and communication overhead, and resisting to packet loss [9]. We consider adopting this approach as the underlying authentication algorithm in this research to take advantage of these desirable merits.

\section{System DESCRIPTION}

The proposed joint authentication and coding system is shown in Fig. 1. At the sender, the multimedia content is firstly passed to the JAC control unit, where it runs the JAC scheme (which will be described in section IV) and outputs the optimal source code rate, channel code rate, and authentication rate. The JSCC unit encodes the multimedia according to both the source rate and outputs the compressed code stream. In the packet signing unit, AG is constructed using the proposed NGBA approach (which will be described in subsection III-A). Therefore, the main task at the sender is to sign and protect the code stream by joint authentication and coding before transmission. At the receiver, error correction is firstly performed on the received stream in the channel decoding unit. Residue errors may still exist in the output stream which passes to the source decoder. We assume that the source decoder is error-resilient, where techniques such as synchronization mark and CRC (cyclic redundancy check) are applied to the code stream. Note that bit errors would trigger verification false alarms, and thus it is important to skip packets 
with bit errors during authentication. The verifiability information passes to the source decoding unit, so that during multimedia decoding, those non-verifiable packets are skipped.

\section{A. Novel Graph-Based Authentication}

In order to obtain lower overhead and higher AP while maintain the same level of delays and robustness against packet loss, we propose a novel graph-based authentication approach where one signature is amortized among a group of packets connected with some regular graphs.

1) Authentication Graph Construction: Assume the stream is divided into a number of blocks and each block contains $M+1(M \geq 0)$ packets, where only one signature is generated for each block, and the $M$ packets and the signature packet $P_{\text {sig }}$ are connected using the regular graph. Assuming $M=n \times m+t(n, m, t \geq 0)$, the definition of the graph is given below.

The $M$ data packets are divided into $m$ stages, and each stage has $n$ packets, and the $t$ is the remaining packets. The packet is denoted as $P(u, v)$, where $u \in\{0,1, \ldots, m-1\}$ indicates the stage and $v \in\{0,1, \ldots, n-1\}$ indicates the packet in a stage. In this graph, there exists a directed edge $e\left(P\left(u_{1}, v_{1}\right), P\left(u_{2}, v_{2}\right)\right)$ from packet $P\left(u_{1}, v_{1}\right)$ to packet $P\left(u_{2}, v_{2}\right)$, if either of the following conditions is met: (1). $u_{1}=u_{2}+1$ and $v_{1}=v_{2}$; (2). $u_{1}=u_{2}+1$ and $v_{1}=v_{2}^{u_{2}}$, where $v_{2}^{u_{2}}$ is different from $v_{2}$ only at the bit position $u_{2}$. In addition, there also exists a directed edge from all packets in stage 0 to the signature packet $P_{\text {sig }}$.

AG CONSTRUCTION

- If there exists $m=\log _{2} n+1$ and $t=0$. Each directed edge $e\left(P\left(u_{1}, v_{1}\right), P\left(u_{2}, v_{2}\right)\right)$ is realized by appending the hash of the packet $P\left(u_{1}, v_{1}\right)$ to $P\left(u_{2}, v_{2}\right)$. Fig. 2(a) gives an example of the authentication graph, with 4 stages and 8 data packets in each stage. The signature packet $P_{\text {sig }}$ contains the signature and hashes of all packets in stage 0 to $\log _{2} n-1$ have two hashes, and the packets in the last stage do not have any hash.

- If there exists $m=\log _{2} n+1$ but $t \neq 0$, the remaining packets $t$ are constructed using the following units (shown in Fig. 3). Note that all packets in stage 0 to $m-1$ have two hashes, and the packets in the last stage (just for the $t$ ) do not have any hash. Fig. 2(b) gives an example of the constructed AG when $M=34=8 \times 4+2$.

2) Lower Bound of AP: For all pairs of nodes $(i, j)$, we include a directed edge from node $i$ to node $j$ with probability $p(0<p \leq 1)$, we call a graph constructed in this way a p-random graph. For notational convenience, we note $P_{\text {sig }}=P_{1}$. 


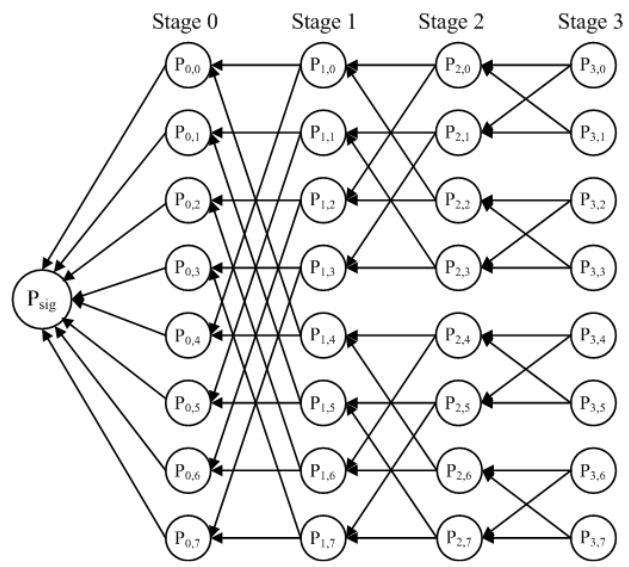

(a) $\mathrm{M}=32$

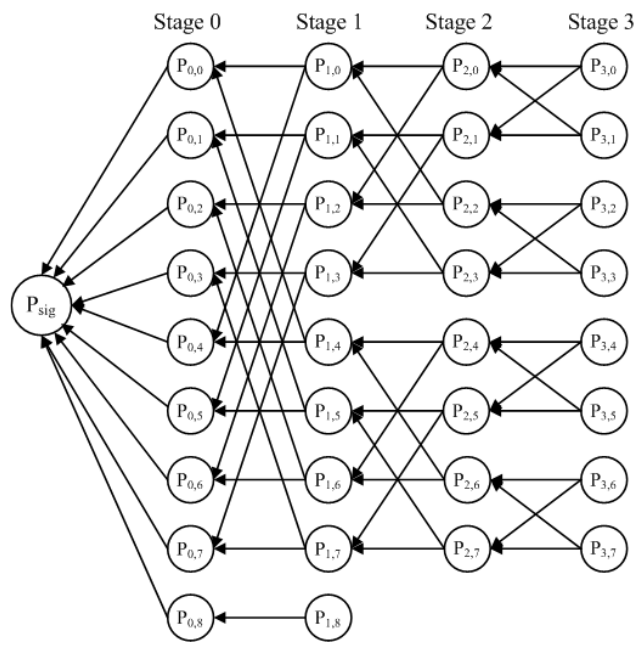

(b) $\mathrm{M}=34$

Fig. 2. The examples of authentication graph

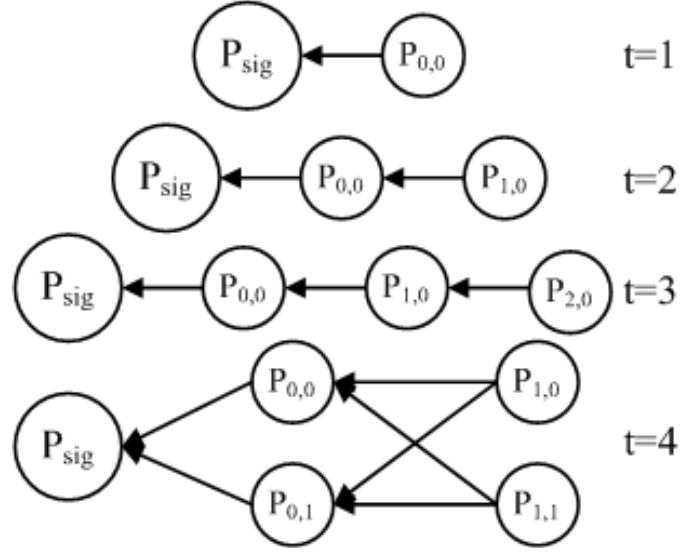

(a) $\mathrm{t}=1,2,3,4$

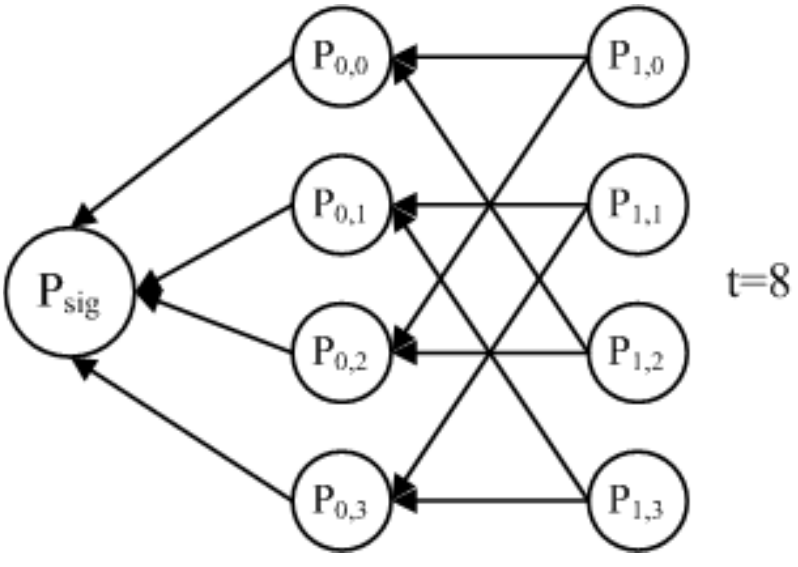

(b) $t=8$

Fig. 3. Basic units of constructing authentication graph.

Lemma 1: With a p-random authentication approach and no packet loss, a packet $P_{i}(i \geq 2)$, can be authenticated with at least the probability:

$$
\operatorname{Pr}\left[P_{i} \rightarrow P_{\text {sig }} \mid P_{i} \text { is received }\right] \geq 1-(1-p)\left(1-p^{2}\right)^{i-2}
$$

Proof: We calculate the probability that node $i$ connects to signature node (node 1 ) in the corresponding p-random graph as follows. First, with probability $p, e(i, 1)$ exists and so, node $i$ connects to the signature node. With probability $(1-p) p, e(i, 1)$ does not exists but $e(i, i-1)$ 
does, so $i$ can connect to signature node via a path from $i-1$ to signature node. Proceeding in this way, we get the following expression:

$$
\begin{aligned}
\operatorname{Pr}\left[P_{i} \rightarrow P_{\text {sig }} \mid P_{i}\right] & \geq p+(1-p) p \operatorname{Pr}\left[P_{i-1} \rightarrow P_{\text {sig }} \mid P_{i-1}\right]+\ldots \\
& +(1-p)^{i-2} p \operatorname{Pr}\left[P_{2} \rightarrow P_{\text {sig }} \mid P_{2}\right]
\end{aligned}
$$

Apply the induction assumption for $1, \ldots, i-1$, to the right hand side of the inequality above, we have:

$$
p+(1-p) p\left(1-(1-p)\left(1-p^{2}\right)^{i-3}\right)+\ldots+(1-p)^{i-2} p(1-(1-p))
$$

We simplify this expression by factoring out terms of the form $(1-p)$. As a first step, we have:

$$
\begin{aligned}
& 1-(1-p)\left[1-p+(1-p) p\left(1-p^{2}\right)^{i-3}-(1-p) p+(1-p)^{2} p\left(1-p^{2}\right)^{i-4}-\ldots\right. \\
& \left.-(1-p)^{i-2} p+(1-p)^{i-3} p\left(1-p^{2}\right)-(1-p)^{i-3} p+(1-p)^{i-2} p\right]
\end{aligned}
$$

Continuing to factor in this way, we eventually get:

$$
\begin{aligned}
& 1-(1-p)^{i-1}\left[p(1+p)^{i-3}+p(1+p)^{i-4}+p(1+p)^{i-5}+\ldots+p(1+p)+1+p\right] \\
= & 1-(1-p)^{i-1}\left[p\left(\frac{1-(1+p)^{i-2}}{1-(1+p)}-1\right)+1+p\right]
\end{aligned}
$$

This simplifies to: $1-(1-p)\left(1-p^{2}\right)^{i-2}$.

Theorem 1: With a p-random authentication approach in a lossy network, in which each packet is lost independently at random with probability $q$, packet $P_{i}, i \geq 2$, can be authenticated with probability:

$$
\operatorname{Pr}\left[P_{i} \rightarrow P_{\text {sig }} \mid P_{i} \text { is received }\right] \geq 1-(1-p)\left(1-(p(1-q))^{2}\right)^{i-2}
$$

Proof: We assume that $P_{1}$ is always received (this may be accomplished with high probability by transmitting it multiple times, or empowering receivers to request re-transmission if it is not received), when we follow the same type of argument as used in the proof of Lemma 1, we get:

$$
\begin{aligned}
\operatorname{Pr}\left[P_{i} \rightarrow P_{1} \mid P_{i}\right] & \geq p+(1-p) p(1-q) \operatorname{Pr}\left[P_{i-1} \rightarrow P_{1} \mid P_{i-1}\right]+\ldots \\
& +(1-p(1-q))^{i-3} p(1-q) \operatorname{Pr}\left[P_{2} \rightarrow P_{1} \mid P_{2}\right]
\end{aligned}
$$




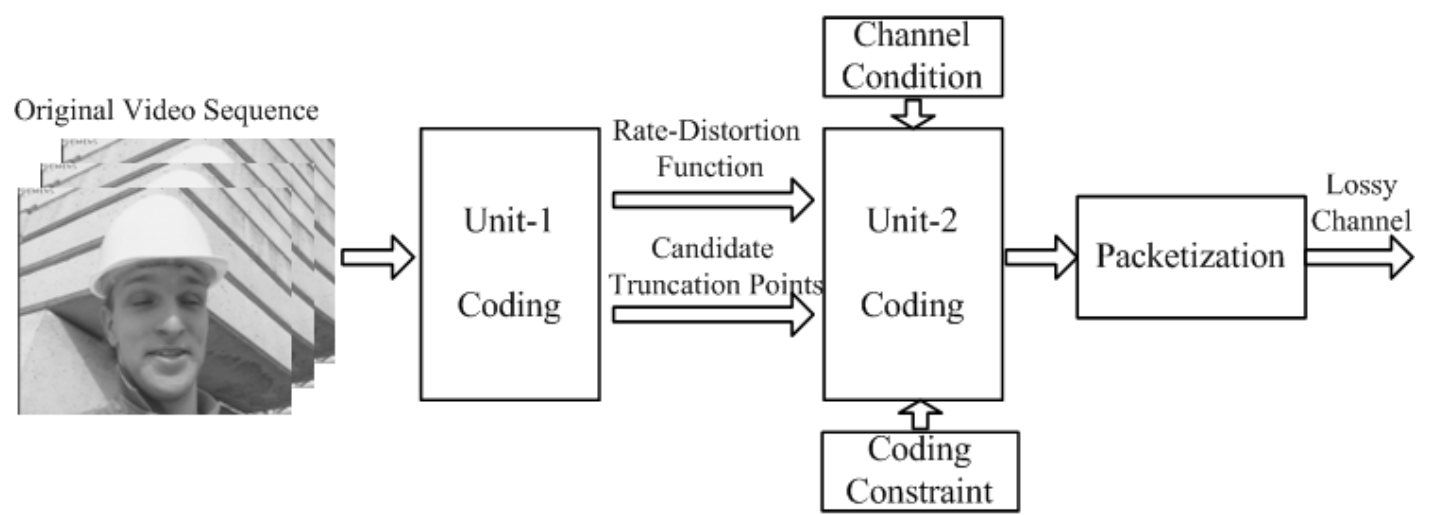

Fig. 4. The architecture of JSCC.

Let $a_{i}(p)=1-(1-p)\left(1-p^{2}\right)^{i-2}$, the authentication probability found in Lemma 1 . From the equality above, it follows that

$$
\operatorname{Pr}\left[P_{i} \rightarrow P_{1} \mid P_{i}\right] \geq\left(\frac{a_{i}(p(1-q))-p}{1-p}\right)(1-p(1-q))+p(1-q)
$$

The statement of the theorem follows from substituting in the expression for $a_{i}(p(1-q))$.

In the case of the proposed NGBA, a packet $P(u, v)$ can not be authenticated unless there is a path to the signature packet at the receiver. The authentication probability $\operatorname{Pr}[\operatorname{P}(u, v)]$ is equivalent to probability that such path exists

$$
\operatorname{Pr}[P(u, v)] \geq 1-(1-p)\left(1-(p(1-q))^{2}\right)^{u}, u \geq 0
$$

We can see that $\operatorname{Pr}[P(u, v)]$ depends only on $u$ and $q$, and all packets in the same stage have the same $\operatorname{Pr}[P(u, v)]$. As we travel from stage 0 to stage $m-1$, the authentication probability decreases, because a packet in the later stage has more independency than that in the earlier stage. However, this trend is slowed down by the proposed graph where a packet in the later stage has more paths to the signature packet. Therefore, the minimum authentication probability $P r_{\text {min }}$ under random packet loss can be achieved as follows

$$
P r_{\text {min }}=1-(1-p)\left(1-(p(1-q))^{2}\right)^{m}
$$

\section{B. Joint Source Channel Coding}

In this subsection, we first introduce the architecture of JSCC, and then describe the packet-loss pattern approximation employed in this paper to represent the channel packet-loss process. 


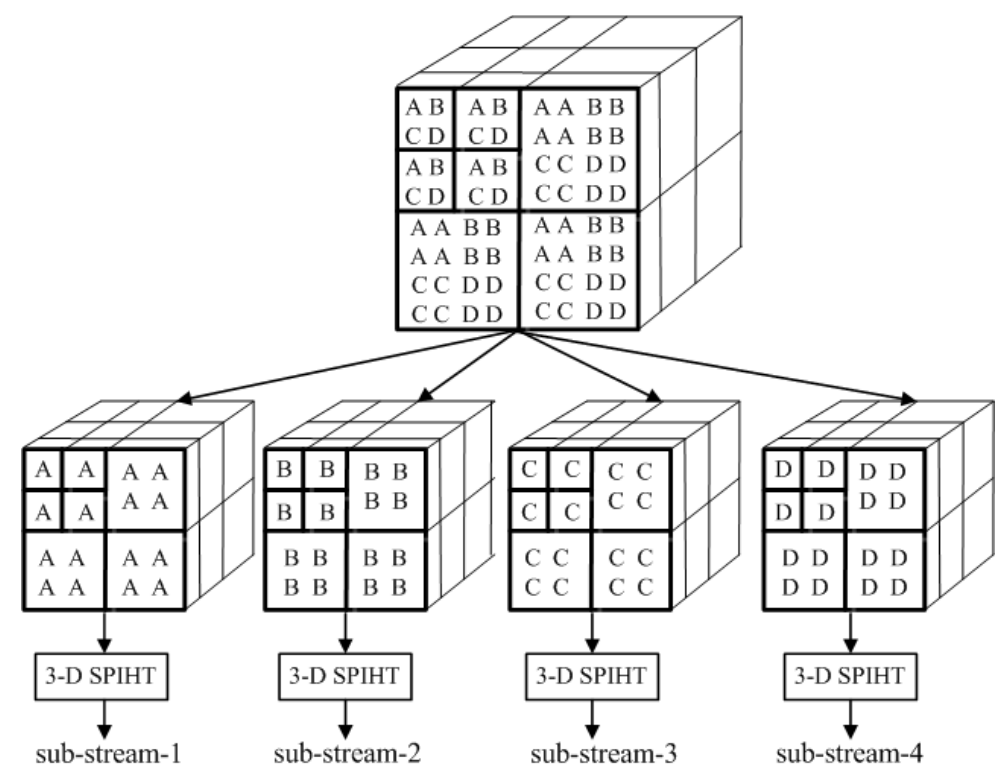

Fig. 5. Separate the 3-D wavelet transform coefficients into four independent sub-streams.

1) The Architecture of JSCC: The proposed coding architecture contains two parts as shown in Fig.4. Unit-1 uses the 3-D SPIHT codec that generates independent embedded streams, while Unit-2 uses the coding constraints and channel condition to pack the bit-streams into packstreams of quality layers. This two-units structure collects incremental contributions from the various streams into SNR scalable quality layers in a way similar to that of embedded block coding with optimized truncation. The streams and rate-distortion functions generated by Unit1 can be processed independently to channel conditions. The source and channel allocation algorithm in Unit-2 must be efficient to cope with the time varying channel conditions.

Unit-1 uses an embedded coding technique that generates multiple independent embedded streams. A video is divided into several independently encoded for additional functionality in Unit-1. The video coder divides the 3-D wavelet coefficients into multiple blocks according to their spatial and temporal relationships, and then encodes each block independently using the 3-D SPIHT algorithm. Fig. 5 shows an example the separation of the 3-D wavelet transform coefficients into four independent blocks, each of which retains the spatio-temporal structure of 3-D SPIHT. The proposed method allocates source bits to each embedded bitstream to minimize the total distortion of a video clip. Moreover, the video scalability is imparted by the layering 
concept and the scalable stream is organized into quality layers.

2) Packet-Loss Pattern Approximation: We use Reed-Solomon (RS) code as the channel coding strategy because it is effective for recovering erased symbols when their locations are known [10]. For a $(n, k)$ systematic RS code with a block length $n$, the source symbol is $k$. The first $k$ encoded symbols are source symbols correctly when the number of loss symbols is less than the minimum distance $d_{\min }=n-k+1$ of the code. The performance of an RS decoder can be characterized by the code correct probability

$$
P_{c}^{\prime}(n, k)=\sum_{m=0}^{n-k} P^{\prime}(n, m)
$$

where $P^{\prime}(n, m)$ is the probability of $m$ erasure within a block of $n$ symbols. In a binary symmetric channel without memory, we have

$$
P^{\prime}(n, m)=\left(\begin{array}{l}
n \\
m
\end{array}\right) P_{B}^{m}\left(1-P_{B}\right)^{n-m}
$$

where $P_{B}$ is the mean packet loss rate [11]. In general, for channels with memory, it is more complicated to calculate. Here, we use a two-state Markov model (i.e. Gilbert model) to simulate the bursty packet loss behavior [12]. The two states of this model are denoted as G (good) and B (bad). In state G, packets are received correctly and timely, whereas, in state B, packets are assumed to be lost. This model can be described by the transition probabilities $P_{G B}$ from state $\mathrm{G}$ to $\mathrm{B}$ and $P_{B G}$ from state $\mathrm{B}$ to $\mathrm{G}$. The then the average $P_{B}$ is given by

$$
P_{B}=\frac{P_{G B}}{P_{G B}+P_{B G}}
$$

The Markov model is a renewal model, and such models are determined by the distribution of error-free intervals, known as gap. Let gap of length $\sigma$ be the event that after a lost packet, $\sigma-1$ packets are received and then again a packet is lost. The gap density function $g(\sigma)$ gives the probability of a gap length $\sigma$. The gap distribution function $G(\sigma)$ gives the probability of the gap length greater than $\sigma-1$. These functions can be derived as [11]

$$
\begin{gathered}
g(\sigma)=\left\{\begin{array}{cc}
1-P_{B G}, & \sigma=1 \\
P_{B G}\left(1-P_{G B}\right)^{\sigma-2} P_{G B}, & \sigma>1
\end{array}\right. \\
G(\sigma)=\left\{\begin{array}{cc}
1-P_{B G}, & \sigma=1 \\
P_{B G}\left(1-P_{G B}\right)^{\sigma-2}, & \sigma>1
\end{array}\right.
\end{gathered}
$$


Let $R(n, m)$ be the probability of $m-1$ erroneous symbols within the next $n-1$ symbols following an erroneous symbol. It can be calculated using the recurrence

$$
R(n, m)=\left\{\begin{array}{cc}
G(n), & m=1 \\
\sum_{\sigma=1}^{n-m+1} g(\sigma) R(n-\sigma, m-1), & 2 \leq m \leq n
\end{array}\right.
$$

Then the probability of errors within $m$ a block of $n$ symbols is

$$
P^{\prime}(n, m)=\left\{\begin{array}{cc}
\sum_{\sigma=1}^{n-m+1} P_{B} G(\sigma) R(n-\sigma+1, m), & 1 \leq m \leq n \\
1-\sum_{\sigma=1}^{n} P^{\prime}(n, m), & m=0
\end{array}\right.
$$

\section{Optimization For Joint Authentication And CODing}

The purpose of joint authentication and coding is to achieve two objectives: (1) optimize the source and channel coding bits for minimizing the end-to-end distortion, and (2) optimize the authentication bits for achieving satisfactory AP. Notice that AP determines the probability that a packet is non-verifiable, which should be skipped during reconstruction. Since the skip will result in distortions to the multimedia content, we may find that it is possible to unify the two objectives into one single form, i.e., maximizing the end-to-end PSNR (Peak Signal-to-Noise Ratio) at the receiver relative to the original sequence. It can be defined as

$$
\operatorname{PSNR}(d B)=10 \log _{10}\left(\frac{255^{2}}{M S E}\right)
$$

where $M S E$ is the mean-square error between the original and the decoded luminance frame.

Fig. 6 shows how multiple encoded sequences of different quality levels are protected based on systematic RS codes. For notational convenience, we define the bit-plane 1 as the highest bit plane and the bit-plane $I_{s}$ as the lowest bit plane to be sent for sub-stream-s [13]. Let $N_{s}$ be the number of packets that are used to send the combined source data and redundancy for sub-stream-s in a GOP (Group of Pictures) and $L$ be the packet size in bytes. In this scheme, the bits belonging to bit-plane $i\left(1 \leq i \leq I_{s}\right)$ are filled into $k_{s, i}$ packets and the remaining $c_{s, i}=N_{s}-k_{s, i}$ packets are filled with channel coding redundancy. In other words, the source data for bit-plane $i$ is protected by $\mathrm{RS}$ code $\left(N_{s}, k_{s, i}\right)$.

We propose the JAC scheme which is performed on GOP basis. We define the total number of packets to be sent from all sources for a GOP period $N$ as 


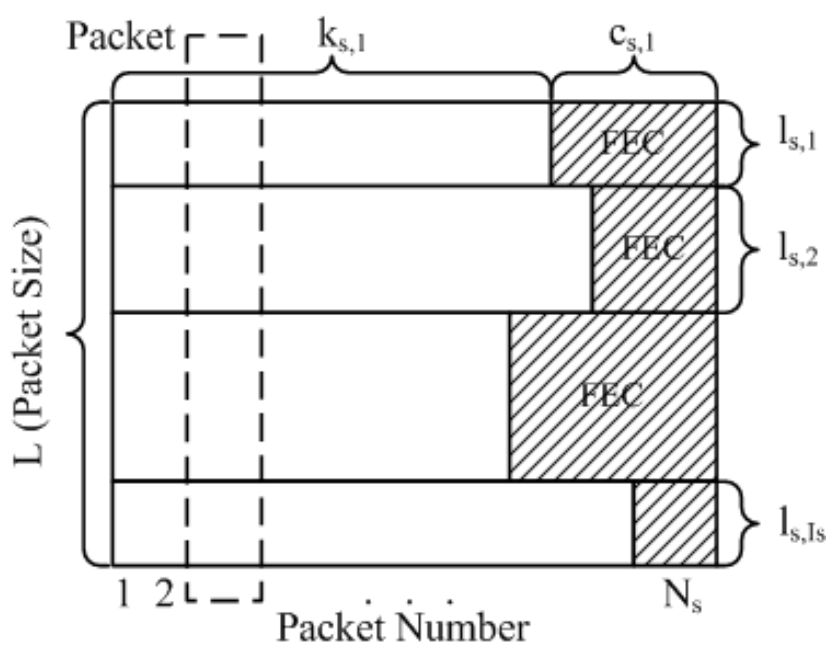

Fig. 6. Multiple-substream bit-plane with error protection.

$$
N \leq\left\lceil\frac{R \times N_{G O P}}{F \times L}\right\rceil
$$

where $R$ is the total coding rate in bytes/s for the combination of source coding $\left(r_{s}\right)$, channel coding $\left(r_{c}\right)$ and authentication $\left(r_{a}\right)$ for all sources, $N_{G O P}$ is the number of frames in a GOP, $F$ is the frame rate in frames/s. In this framework, we assume that are $n_{s}$ sources and source-s transmits sub-stream-s to the receiver for $s=1,2, \ldots, n_{s}\left(n_{s} \geq 1\right)$. Then the proposed algorithm divides $N$ into $N_{1}\left(t_{i}\right), N_{2}\left(t_{i}\right), \ldots, N_{n_{s}}\left(t_{i}\right)$ so as to maximize the expected quality at the receiver, where $N_{s}\left(t_{i}\right)$ represents the total number of packets transmitted by source-s at GOP period $t_{i}$ for $s=1,2, \ldots, n_{s}\left(n_{s} \geq 1\right)$. Taking account into the effective rate of source $s, N_{s}\left(t_{i}\right)$ should satisfy the following condition:

$$
N_{s}\left(t_{i}\right) \leq\left\lceil\frac{R_{s}\left(t_{i}\right) \times N_{G O P}}{F \times L}\right\rceil
$$

where $R_{s}\left(t_{i}\right)$ is the total rate of source-s at the GOP period $t_{i}$. In typical transform coding, each coefficients is quantized independently. The overall distortion is exactly the summation of the distortion at each source. The probability for an authentic packet $P_{i}$ to be decodable and verifiable is $\operatorname{Pr}_{i}\left(1-P_{B}\right)$. In this case, the distortion is merely due to source coding. If the packet is either non-decodable or non-verifiable, the distortion depends on the specific errorconcealment scheme. Here, we consider setting the values to zeros when a packet is either non- 
decodable or non-verifiable. Therefore, we can state our source and channel allocation algorithm as follows: Given $N, R_{s}\left(t_{i}\right)$ and the tolerated minimum authentication probability $P r_{t h r}$, the proposed algorithm finds $N_{s}\left(t_{i}\right)$ and $K_{s}\left(t_{i}\right)=\left(k_{s, 1}\left(t_{i}\right), k_{s, 2}\left(t_{i}\right), \ldots, k_{s, I_{s}}\left(t_{i}\right)\right)$ for $s=1,2, \ldots, n_{s}$, that maximize the expected quality at the receiver given by

$$
\begin{gathered}
P S N R\left(t_{i}\right)=\sum_{s=1}^{n_{s}}\left(\operatorname{Pr}_{s}\left(1-P_{B}\right)\right) \sum_{l=1}^{I_{s}}\left(\sum_{j=N_{s}-k_{s, l}+1}^{N_{s}-k_{s, l-1}} P^{\prime}\left(j, N_{s}\right) \sum_{i=l}^{I_{s}} P S N R_{s}(i)\right) \\
\text { subject to } \sum_{s=1}^{n_{s}} N_{s}=N, \\
N_{s} \leq\left\lceil\frac{R_{s}\left(t_{i}\right) \times N_{G O P}}{F \times L}\right\rceil \\
\operatorname{Pr}_{s} \geq \operatorname{Pr}_{t h r}, s=1,2, \ldots, n_{s}
\end{gathered}
$$

where $\operatorname{Pr}_{s}$ is the average AP of source-s; $P^{\prime}\left(j, N_{s}\right)$ is the probability that $j$ packets are lost out of $N_{s}$ packets sent by source-s; $P S N R_{s}(i)$ is the expected quality at the receiver when the receiver decodes up to the $i$ th bit-plane for sub-stream-s; $I_{s}$ is the last bit plane to be sent for source-s.

Each source independently runs the proposed rate allocation algorithm to get its optimal number of packets to transmit for a GOP period, using the information contained in the control packets that the receiver sends to all sources. The proposed algorithm tries all possible combinations of $\left(N_{s}, K_{s}\right)$ that satisfy the constraints in (21) and choose one that maximizes the expected quality. Once the optimal $\left(r_{s}, r_{c}\right)$ value is found, the source code rate, channel code rate and authentication rate are determined.

\section{Simulation Results and Discussion}

We conducted simulation experiments to test the performance of the proposed streaming framework. First of all, we describe the simulation environment. Secondly, we present the main simulation results where we show the objective and subjective results of the performance under different scenarios. Finally, we conclude this section based on the selected simulation results described.

\section{A. Simulation Environment}

For these experiments, we use the QCIF Weather Forecast test sequence at $F=30 \mathrm{frames} / \mathrm{s}$, $N_{G O P}=16$ and $n_{s}=2$. A three-level wavelet decomposition is applied to a group of 16 frames 
TABLE I

COMPARISON WITH COMPETING APPROACHES

\begin{tabular}{|c|c|c|c|}
\hline & Communication Overhead & Receiver Delay & Maximum Burst Loss \\
\hline Random Graph [5] & $\mathrm{M}, 1$ & 1 & Unconsidered \\
\hline EMSS [3] & $\mathrm{M}, 1$ & $\mathrm{M}$ & $\mathrm{b}-1$ \\
\hline Erasure Code [6] & $\mathrm{M}, 1,2$ & {$\left[\mathrm{~m}^{\prime}, \mathrm{M}\right]$} & $\mathrm{M}-\mathrm{m}^{\prime}$ \\
\hline NGBA & $\mathrm{M}, 1$ & 1 & $n / 2^{m+1}$ \\
\hline
\end{tabular}

and the 3-D wavelet coefficients are divided into two groups using the method proposed in [10].

In order to provide a representative evaluation of system performance, for each simulation run we generate a random topology on the disc of unit area as a 2D Poisson point process with total number of nodes equal to 25. The transmission range $r$ for each node is kept constant during the simulation at the value of $r=0.2 \times(1 / \sqrt{\pi})$ such that the sum of the transmission regions for all the 25 nodes (i.e., $25 \times \pi r^{2}=1$ ) almost completely covers the unit disc, thus ensuring a high degree of connectivity. Each node is assigned the fixed transmission rate $W_{i}=2 \mathrm{Mbps}$, which is a basic rate available in the IEEE 802.11 b standard. During transmission, the environments are updated every 1 second which can cause changes in the channel condition. During successive 1 second intervals, the environments are kept constant. It should be noted that all the simulation results in this section have been obtained using averaged 300 runs in order to obtain statistically meaningful results.

\section{B. Selected Simulation Results and Discussions}

At first, we compare the proposed NGBA approach with other existing approaches. Table I summarizes the 4 authentication approaches based on aforementioned requirements. (Note: $M$ is the block size; $\mathrm{b}$ is the maximum edge length; $m^{\prime}$ is the minimum number of received packets to recover the hashes and the signature in a block; $\mathrm{m}$ is the number of the stage; $\mathrm{n}$ is the number of packets each stage contains)

In most approaches, the authentication probability and communication overhead conflict with each other, that is, increasing the overhead will increase the authentication probability, and vice versa. Fig. 7 shows the authentication probabilities under different communication overheads. Assuming the loss probability is $30 \%$, the total number of packet is 1024 , each hash has 16 bytes 


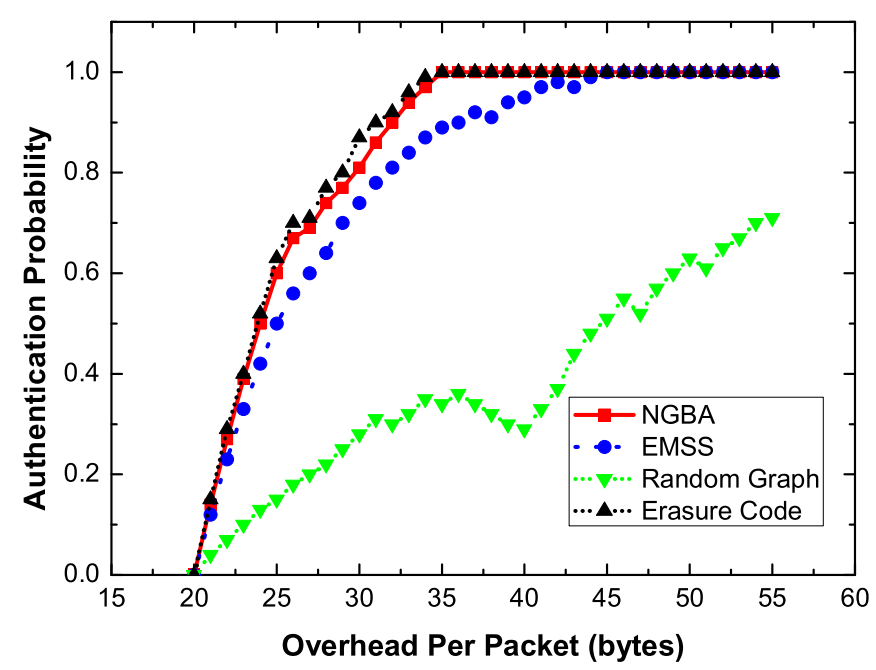

Fig. 7. Authentication probabilities at different overheads. (Packet loss probability is fixed at 30\%)

and each signature has 128 bytes. For EMSS approach, the length of each edge is uniformly distributed in the interval [1,128]; Fig. 8 shows that our proposed approach outperforms other approaches except the Erasure Code in terms of overhead and authentication probability. From the above figures, we can see that the NGBA outperforms existing approaches in terms of integrating overhead, robustness, authentication probability and receiver delay.

And then, to demonstrate the effectiveness of our proposed joint scheme, we plot the endto-end rate-distortion curves for the test sequence at packet loss rate equal to $5 \%$ and $15 \%$, respectively. The proposed resource allocation scheme (JAC+NGBA) is benchmarked against other two schemes:1) JAC+EMSS, in which the overall resource allocation is performed between source channel coding and authentication, but the resource within authentication is equally allocated using the basic EMSS scheme. 2) JSCC+EMSS, in which the resource for source and channel coding is jointly allocated whereas that for authentication is fixed, and the basic EMSS is applied. Fig. 9 shows the performance comparison between our proposed scheme and the competing schemes. The proposed JAC+NGBA scheme can be seen to achieve a much higher performance in terms of end-to-end PSNR compared to the competing schemes. When the packet loss rate is 5\% and the overall rate ranges from 0.5 to 3 , the average PSNR using the 


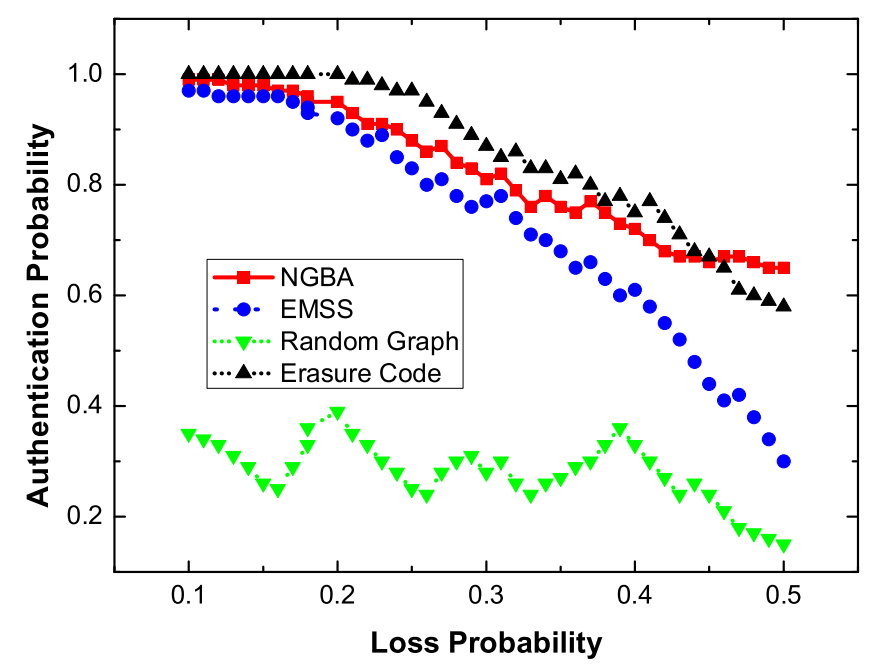

Fig. 8. Authentication probabilities at different loss probabilities. (The overheads is 30 bytes per packet)

proposed scheme is $37.85 \mathrm{~dB}$ while it is $34.60 \mathrm{~dB}$ and $34.26 \mathrm{~dB}$ for the case of JAC+EMSS and JSCC+EMSS, thus, around 3.2-3.6 dB performance gain can be achieved on average using the proposed scheme. Similarly, when the packet loss rate is $15 \%$, around $2.6-5.6 \mathrm{~dB}$ performance gain can be achieved on the average. It should be noted that JAC+EMSS also outperforms JSCC+EMSS, especially when the packet loss rate is high. For example, when the packet loss rate is $5 \%$, the average performance gap is only $0.34 \mathrm{~dB}$; while packet loss rate is $15 \%$, the gap increases to $3.05 \mathrm{~dB}$.

Moreover, to examine how the JAC is affected by the channel condition, we fix the overall code rate and examine how $r_{s}, r_{c}$ and $r_{a}$ vary, as the packet loss rate increases from 5\% to $15 \%$. Table II illustrates the unitary results for the test sequence. From the table, we observe that when the channel condition is good, most of the bits are allocated for source coding and authentication. When the channel condition is poor, the large portion of bits is allocated for channel coding. As expected, the PSNR of reconstructed image decreases as packet loss rate increases.

The above objective results are based on a quantitative assessment of reconstructed PSNR values. In Fig. 10, we also show some subjective results based on the reconstructed frames 


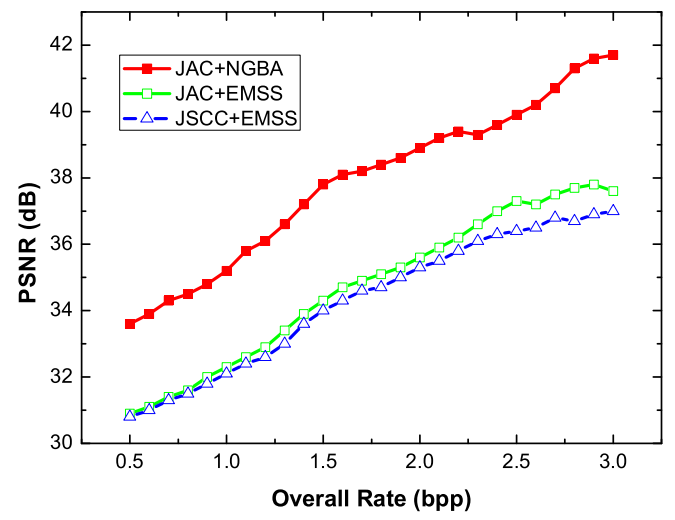

(a)

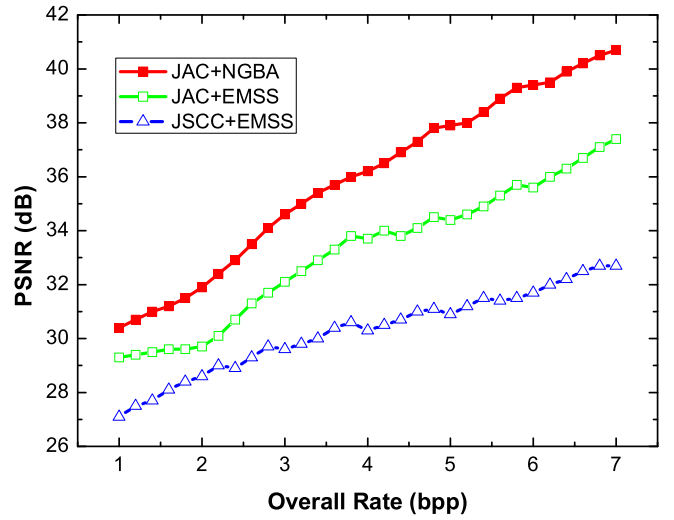

(b)

Fig. 9. End-to-end rate-distortion curves. (a) packet loss rate is 5\% ( $r_{a}=0.4$ for JSCC+EMSS). (b) packet loss rate is $15 \%$ $\left(r_{a}=0.25\right.$ for JSCC+EMSS).

TABLE II

JAC RATE UNDER DIFFERENT $P_{B}$ (OVERALL RATE=3BPP)

\begin{tabular}{|c|c|c|c|c|c|c|c|c|c|c|c|}
\hline$P_{B}$ & $5 \%$ & $6 \%$ & $7 \%$ & $8 \%$ & $9 \%$ & $10 \%$ & $11 \%$ & $12 \%$ & $13 \%$ & $14 \%$ & $15 \%$ \\
\hline$r_{s}$ & 0.54 & 0.52 & 0.51 & 0.48 & 0.46 & 0.41 & 0.38 & 0.35 & 0.33 & 0.29 & 0.25 \\
\hline$r_{c}$ & 0.04 & 0.13 & 0.16 & 0.21 & 0.24 & 0.31 & 0.38 & 0.42 & 0.48 & 0.53 & 0.59 \\
\hline$r_{a}$ & 0.42 & 0.35 & 0.33 & 0.31 & 0.30 & 0.28 & 0.24 & 0.23 & 0.19 & 0.18 & 0.16 \\
\hline PSNR(dB) & 41.7 & 41.1 & 40.6 & 40.1 & 39.3 & 38.8 & 37.8 & 36.9 & 36.0 & 35.4 & 34.6 \\
\hline
\end{tabular}

taken from the decoded test sequence of the simulation run when $P_{B}=15 \%$ and the overall rate is 4 bpp. From Fig. 10, we can see that the proposed JAC+NGBA scheme can provide improved subjective performance compared to the other competing schemes. These results again support the preceding objective assessments.

In order to provide a more comprehensive evaluation of the proposed joint scheme, in Table III, we repeat the results for other QCIF video sequences under the same simulation configuration as the previous experiments (Note: $r_{a}=0.3$ for JSCC+EMSS). From Table III, it can be observed that the proposed JAC+NGBA method has considerable performance advantage comparing to the other competitive methods, which is due to the proposed scheme has the characteristics of 


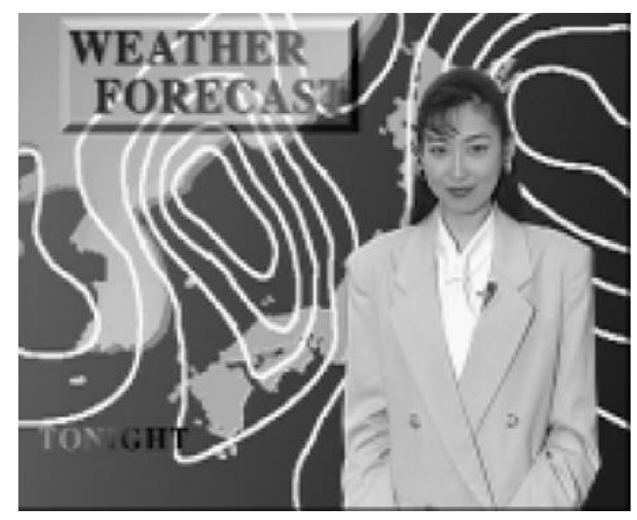

(a)

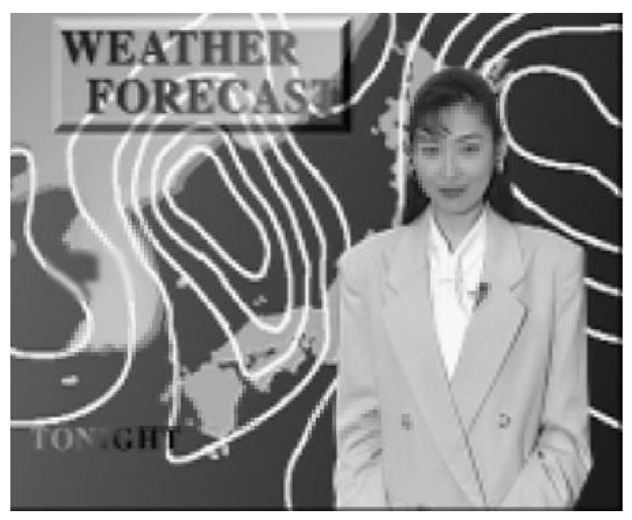

(b)

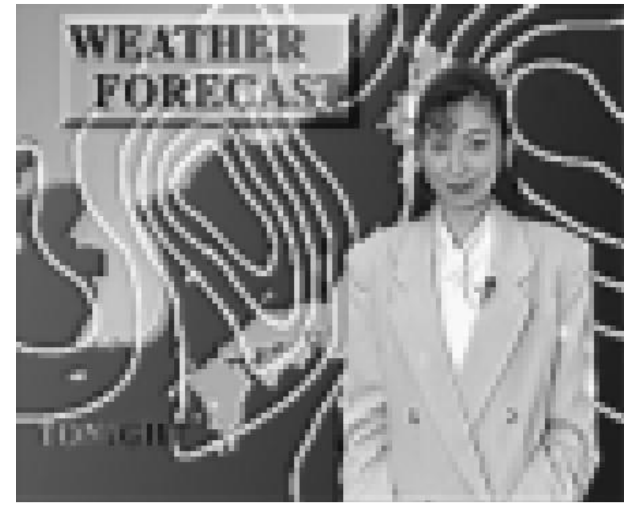

(c)

Fig. 10. Subjective comparisons of decoded frames for Weather Forecast sequence. (a) Joint JAC and NGBA; (b) Joint JAC and EMSS; (c) Joint JSCC and EMSS; (PSNR=36.7 dB, $34.2 \mathrm{~dB}, 30.8 \mathrm{~dB}$, respectively).

network-adaptive and error-resilient.

\section{CONCLUding REMARKS}

In this paper, we have been focusing on designing a joint authentication and coding system in order to achieve satisfactory authentication results and end-to-end reconstruction quality under the overall limited resource budget. Firstly, we provide a novel graph-based authentication approach which can not only construct the authentication graph flexibly but also trade-off well between some practical requirements. Secondly, we propose an analytical joint source-channel coding approach for error-resilient scalable encoded video for lossy transmission. Furthermore, we integrate authentication with coding to achieve an optimal end-to-end multimedia quality under 
TABLE III

PERFORMANCE COMPARISON FOR OTHER SEQUENCES UNDER DIFFERENT SIMULATION CONDITIONS

\begin{tabular}{c||c||c||c|c|c}
\hline \multirow{2}{*}{ Video } & \multirow{2}{*}{$\begin{array}{c}\text { Packet Loss } \\
\text { Sequence }\end{array}$} & Overall & \multicolumn{3}{c}{ PSNR of different methods (dB) } \\
\cline { 4 - 6 } Stefan & Rate & Rate (bpp) & JAC+NGBA & JAC+EMSS & JSCC+EMSS \\
\hline \multirow{2nnnyy}{*}{ Football } & $5 \%$ & 1.5 & 35.3 & 31.7 & 31.1 \\
& $15 \%$ & 3 & 32.5 & 29.3 & 27.2 \\
\hline \multirow{2}{*}{ Coastguard } & $5 \%$ & 1.5 & 34.6 & 30.1 & 30.0 \\
& $15 \%$ & 3 & 31.2 & 30.5 & 29.7 \\
\hline \multirow{2}{*}{ Calendar } & $5 \%$ & 1.5 & 36.8 & 33.2 & 32.4 \\
& $15 \%$ & 3 & 34.7 & 31.5 & 31.3 \\
\hline \multirow{2}{*}{ Mobile } & $5 \%$ & 1.5 & 35.9 & 32.0 & 31.4 \\
& $5 \%$ & 3 & 32.8 & 30.1 & 30.1 \\
\hline Foreman & $5 \%$ & 1.5 & 34.1 & 32.6 & 31.8 \\
& $15 \%$ & 3 & 31.7 & 30.8 & 29.9 \\
\hline
\end{tabular}

the overall limited resource budget. The simulation results show the effectiveness of our joint authentication-coding scheme for multimedia over wireless networks.

\section{ACKNOWLEDGMENTS}

This work is supported by the International Project PRA-SI (financed by France and China government) under Grant No. SI04-03, the Key Project of Nature Science Foundation of Jiangsu (China) under Grant BK2007729 and the Climbing Plan in NJUPT under Grant NY207061. Moreover, we also thank the anonymous reviewers for insightful comments and suggestions. 


\section{REFERENCES}

[1] Zhishou Zhang, Qibin Sun, Wai-choong Wong et al. An Optimal Content-Aware Authentication Scheme for Streaming JPEG-2000 Images over Lossy Networks. IEEE Tran. Multimedia; 2007; 9(2), pp. 320-331.

[2] R. Gennaro, P. Rohatgi. How to Sign Digital Streams. In Advances in Cryptology-CRYPTO'97

[3] A. Peffig, R. Canetti, J. Tygar et al. Efficient Authentication and Signing of Multicast Streams over Lossy Channels. Proc. of IEEE Symposium on Security and Privacy; 2000; pp.56-73.

[4] D. Song, D. Zuckerman, J. Tygar. Expander Graphs for Digital Stream Authentication and Robust Overlay Networks. Proc. of IEEE Symposium on Research in Security and Privacy; 2002; pp. 258-270.

[5] S. Miner, J. Staddon. Graph-Based Authentication of Digital Streams. Proc. of IEEE Symposium on Research in Security and Privacy; 2001; pp. 232-246.

[6] J. M. Park, E. K. P. Chong, and H. J. Siegel. Efficient Multicast Stream Authentication using Erasure Codes. ACM Trans. Inf. Syst. Secur.; 2003; 6(2), pp.233-245.

[7] Zhang, Z., Sun, Q., Wong, W.-C et al. Rate-Distortion-Authentication Optimized Streaming of Authenticated Video. IEEE Trans. Circuits and Systems for Video Technology; 2007; 17(5), pp.544-557.

[8] Lysyanskaya, Anna. Authentication without Identification. IEEE Security \& Privacy Magazine; 2007; 5(3), pp. 69-71.

[9] Li, Z., Sun, Q., Lian, Y., et al. Joint Source-Channel-Authentication Resource Allocation and Unequal Authenticity Protection for Multimedia over Wireless Networks. IEEE Trans. Multimedia; 2007; 9 (4), pp. 837-850.

[10] Joohee Kim, Russel M.Mersereau, Yucel Altunbasak. Distrubuted Video Streaming Using Multiple Description Coding and Unequal Error Protection. IEEE Transactions on Image Processing; 2005; 14(7), pp. 849-861.

[11] Chi-Ming Fu, Wen-Liang Hwang, et al. A Joint Source and Channel Coding Algorithm for Error Resilient SPIHT-Coded Video Bitstreams, Journal of Visual Communication \& Image Representation; 2006; 17, pp. 1164-1177.

[12] Qi Qu, Yong Pei, et al. Cross-layer QoS Control for Video Communications over Wireless Ad Hoc Networks. EURASIP Journal on Wireless Communications and Networking, 2005; 5, pp. 743-756

[13] Sungdae, Cho Pearlman, W. A. A full-featured, error-resilient, scalable wavelet video codec based on the set partitioning in hierarchical trees (SPIHT) algorithm. IEEE Transactions on Circuits and Systems for Video Technology, $2002 ; 12$ (3), pp. 157-171.

[14] Yongdong Wu, Robert H. Deng. Scalable Authentication of MPEG-4 Streams. IEEE Trans. on Multimedia, 2006; 8 (1), pp.152-161. 